\title{
New diversity in the sulfur cycle
}

Microorganisms perform key functions in the global sulfur cycle, such as sulfate reduction and the synthesis of organic sulfur molecules. Two studies identify new contributions to the microbial sulfur cycle; one study shows that the capacity for dissimilatory sulfite and sulfate reduction is more common than previously thought and the other identifies the key enzyme that is responsible for the production of dimethylsulfoniopropionate (DMSP) in phytoplankton.

Sulfate-reducing bacteria and archaea can use sulfate instead of oxygen as the terminal electron acceptor. This form of anaerobic respiration produces hydrogen sulfide and was essential for microbial

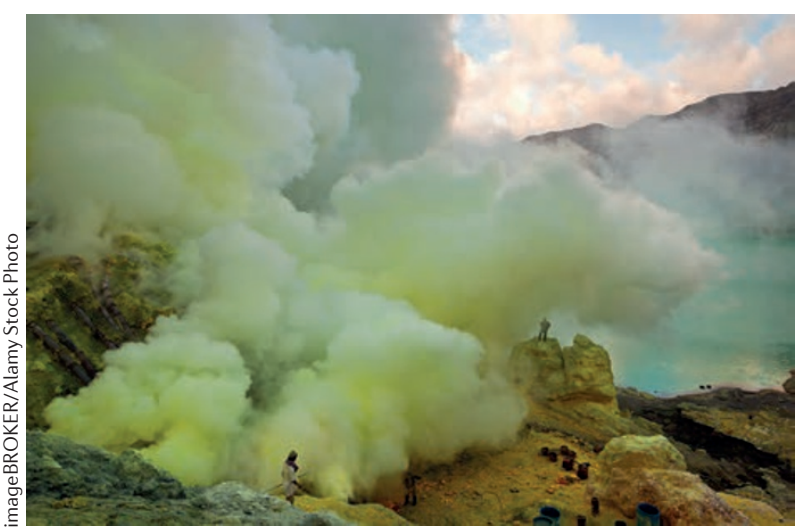

energy generation on the early Earth, before the great oxygenation event. Sulfate reduction can also be coupled to the oxidation of organic carbon, which makes it an important component of the carbon cycle in environments such as marine and terrestrial sediments and wetlands. Previously, novel dissimilatory sulfite reductase genes, $d s r A$ and $d s r B$, had been identified; however, it was unclear in which organisms they occurred. Anantharaman et al. screened 4000 near-complete metagenome-assembled genomes for the presence of sulfite reductase genes, including $d s r A$ and $d s r B$. They found that 20 bacterial and archaeal phyla had members that encode sulfite reductases, which more than doubles the number of phyla with this metabolic capacity. Interestingly, the $d s r$ genes were transferred horizontally between phyla several times and have a complex evolutionary history. The authors conclude that dissimilatory sulfur metabolism is more widespread than previously thought and occurs in microorganisms that inhabit diverse environments.

The second study set out to identify the enzyme that is responsible for the synthesis of DMSP, which is an osmolyte and an important sulfur source in marine environments. DMSP is mainly produced by eukaryotic phytoplankton, although some bacteria also can produce it. Recently, DsyB had been identified as the key enzyme for the synthesis of DMSP in bacteria; however, its eukaryotic counterpart was unknown. Curson et al. found a homologous gene, which they termed $D S Y B$, in many eukaryotes, including dinoflagellates, haptophytes, diatoms and corals, and confirmed the DMSP-biosynthetic activity of the encoded enzymes. They showed that DSYB is located in the chloroplasts and mitochondria of the eukaryotic algal species Prymnesium parvum and, based on immunolabelling and stable-isotope tracking, they suggest that DMSP synthesis occurs in these organelles and might protect them from reactive oxygen species.

Diverse microorganisms contribute to the global sulfur cycle through various reactions, as shown in these two new studies that identified the bacterial and archaeal taxa that can perform dissimilatory sulfur reduction and a key enzyme for DMSP production in phytoplankton, respectively.

Ursula Hofer

ORIGINAL ARTICLES Anantharaman, K. et al. Expanded diversity of microbial groups that shape the dissimilatory sulfur cycle. ISMEJ. https://doi. org/10.1038/s41396-018-0078-0 (2018) | Curson, A. R. et al. DSYB catalyses the key step of dimethylsulfoniopropionate biosynthesis in many phytoplankton. Nat Microbiol. https://doi.org/ 10.1038/s41564-018-0119-5 (2018) 